\title{
High-content analysis of biomarker intensity and distribution in 3D microtissues
}

\author{
Three-dimensional (3D) cell culture methods are widely accepted as more physiologically relevant than \\ conventional 2D cell culture methods and are believed to improve the prediction of drug candidates \\ at an early stage of the drug development process. Here we describe the analysis of a spherical colon \\ cancer microtissue model using the Operetta ${ }^{\circledR}$ High Content Imaging System. In vivo near-infrared \\ (NIR) agents allowed visualization and quantification of cancer-associated biomarker intensity and \\ distribution in microtissues.
}

\begin{abstract}
The Operetta ${ }^{\circledR}$ High Content Imaging System is an automated microscope capable of acquiring fluorescence and bright-field images from samples in microplates or on slides. The system is equipped with a spinning-disk confocal scanner that is well suited for confocal imaging of 3D cell models such as spherical microtissues. Performing image acquisition as well as image analysis and data management, the Harmony ${ }^{\circledR}$ High Content Imaging and Analysis Software provides an intuitive user interface that allows a step-by-step approach to image analysis.

PerkinElmer in vivo NIR agents are designed to monitor and quantify biological events such as cancer or inflammatory diseases in small animals. The NIR agents are composed of two parts: the biological part mediates the interaction with the target molecule or gets cleaved by a target protein, and an NIR dye with an emission wavelength of $700 \mathrm{~nm}$ visualizes the biological event.
\end{abstract}

Quantifying cancer biomarker expression with in vivo NIR agents

Assay-ready live tumor microtissues consisting of HT-29 colorectal adenocarcinoma cells were provided by InSphero AG. The microtissues were shipped in 96-well GravityTRAPTM plates that allowed easy localization of tissues in the wells during imaging on the Operetta system ${ }^{1}$. To analyze the activity of the cancer-associated biomarkers cathepsin and matrix metalloproteinase (MMP) ${ }^{2-4}$, and to visualize hypoxic areas ${ }^{5}$, microtissues were stained with $100 \mathrm{nM}$ of the NIR agents ProSense ${ }^{\circledR} 680$ (NEV10003), MMPSense ${ }^{\circledR} 680$ (NEV10126) and HypoxiSense ${ }^{\circledR} 680$ (NEV11070), respectively. Images were acquired $72 \mathrm{~h}$ after staining at a focus height of $20 \mu \mathrm{M}$

Marcel Waschow ${ }^{1}$, Stefan Letzsch ${ }^{1}$, Karin Boettcher ${ }^{1}$ \& Jens Kelm ${ }^{2}$

${ }^{1}$ PerkinElmer, Cellular Technologies Germany GmbH, Hamburg, Germany. ${ }^{2}$ Insphero AG, Zurich, Switzerland. Correspondence should be addressed to K.B. (karin.boettcher@ perkinelmer.com) in confocal mode with the $10 \times$ high-numerical aperture objective. The ProSense 680 agent homogeneously stained the microtissue, which corresponds to observations in vivo in which the agent stained all cells in the tumor homogeneously. The MMPSense 680 agent was mainly activated in isolated cells. MMPs are known to play a crucial role in cancer cell migration and metastasis ${ }^{6}$. The activation of MMPSense agent in isolated cells suggests the contribution of MMP activity to the degradation of extracellular matrix and escape of these cells from the tissue. The HypoxiSense 680 agent showed the strongest signals in the core region of microtissues, which clearly indicates the presence of an oxygen gradient toward the core region (Fig. 1).

To quantify the different labeling phenotypes, microtissues were segmented using the Harmony software's 'Find Nuclei' building block, based on the Hoechst 33342 channel. In order to quantify regional agent intensities, the segmented microtissues were subdivided into a core region, a surrounding core region and a

Fluorescense +
bright-field
image
Fluorescense
image
staining patterns. Microtissues were incubated with 100 nM of the near-
infrared (NIR) agents for $72 \mathrm{~h}$. The top row shows an overlay of bright-field
and fluorescence images, and the lower row shows only the fluorescence
images. The ProSense 680 agent (left) shows a homogeneous staining of the
whole microtissue. The MMPSense 680 agent (middle) is strongly activated in
isolated cells and shows a weak fluorescence signal within the microtissue. The
HypoxiSense 680 agent (right) stains the microtissue with fluorescence maxima
in the core region.



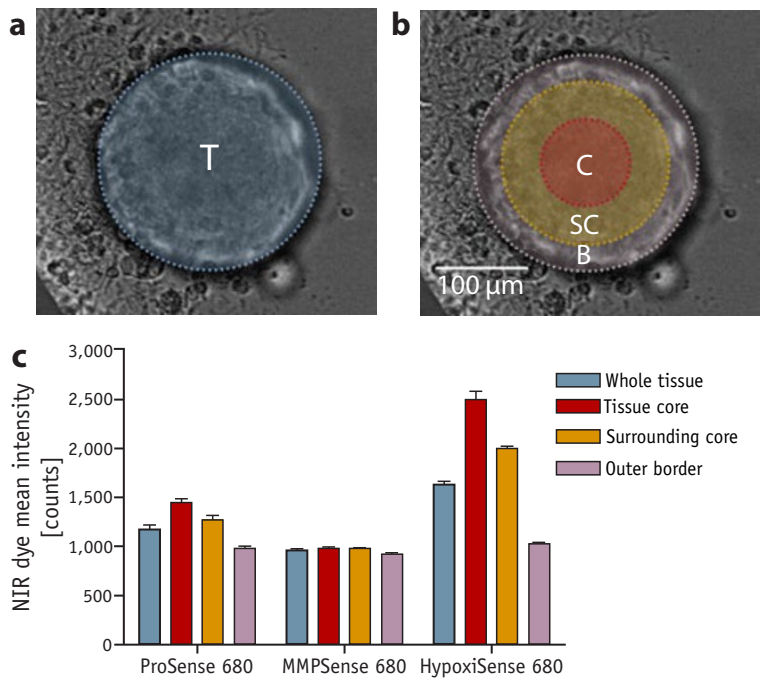

Figure 2 | Quantitative analysis of regional intensities of the in vivo near-infrared (NIR) agents in microtissues. Microtissues were subdivided into different regions (whole tissue, tissue core, surrounding core and outer border) to quantify the different staining patterns. (a) Whole tissue area ( $T$, blue). (b) Microtissue with indicated outer border (B, gray), surrounding core (SC, yellow) and tissue core region ( $C$, red). (c) Mean fluorescence intensities of ProSense 680, MMPSense 680 and HypoxiSense 680 agents in the different regions ( $n=3$ per dye).

border region using the 'Select Region' building block of the Harmony software (Fig. 2a,b). The regional agent intensities were calculated using the 'Calculate Intensity Properties' building block and compared with the intensity of the whole microtissue region (Fig. 2c).

\section{Studying hypoxic conditions in microtissues}

To study hypoxic conditions, microtissues of varying sizes were produced by seeding different cell numbers during spheroid formation. Microtissues were stained with HypoxiSense 680 agent and imaged on the Operetta system, and the maximum intensity in the spheroid core region was analyzed using the Harmony software. The maximum intensity is positively correlated with the size of the microtissues. Larger microtissues show a stronger increase in the core maximum intensity than smaller microtissues (Fig. 3). The hypoxia gradient toward the core can be attributed to a limited penetration of oxygen and potentially other nutrients into the tissue, as has been described for avascular tumors in vivo ${ }^{7}$.

\section{Conclusions}

The high-content analysis of 3D microtissues with in vivo NIR agents resulted in a successful quantification of disease-associated cancer biomarkers in microtissues. The automated confocal imaging of spheroids on the Operetta system and the intuitive building block image analysis of the Harmony software provided an ideal tool to characterize 3D microtissues. The characteristic labeling pattern of the NIR agents is observed in a similar way in vivo and confirms microtissues to be a physiologically relevant cell model that resembles solid tumors. Due to their long excitation $(680 \mathrm{~nm})$ and emission (700 nm) wavelengths, the NIR agents are especially useful for studying microtissues because light absorption and scattering in
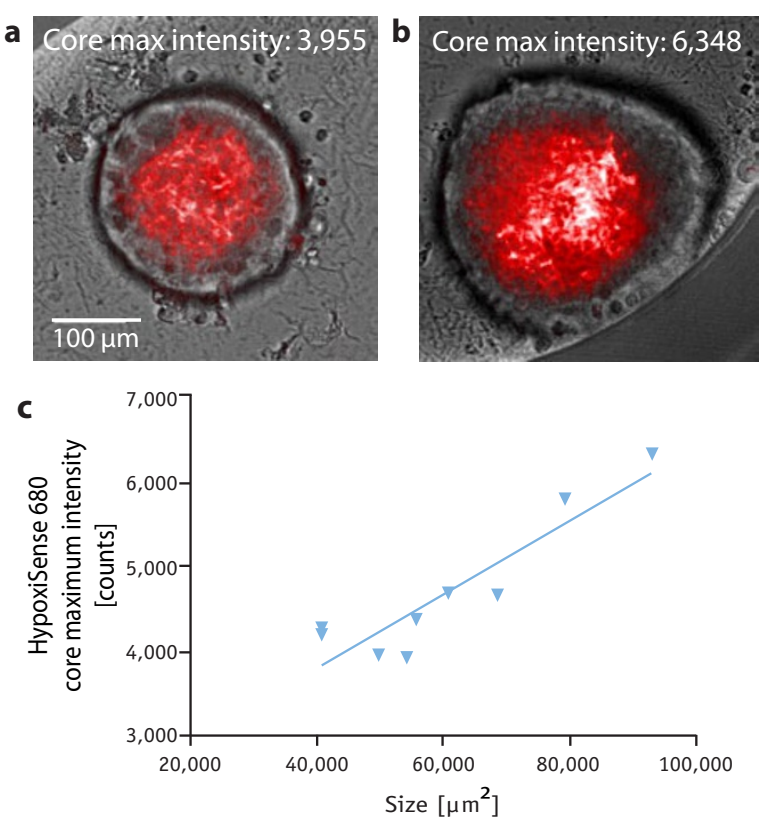

Figure $\mathbf{3}$ | HypoxiSense 680 agent staining intensity of microtissues is size dependent. (a) Overlay of bright-field and fluorescence images of two representative microtissues. The larger microtissue on the right (area =93,197 $\mu^{2}$ ) shows a strongly increased HypoxiSense maximum signal intensity compared with the smaller tissue on the left $\left(\right.$ area $\left.=49,844 \mu \mathrm{m}^{2}\right)$. (b) With increasing size the microtissue cores show a strong increase in maximum signal intensity. This suggests the presence of small hypoxic centers.

tissue is very low at these wavelengths. The possibility of using in vivo NIR agents as translational tools in biochemical, cellular and in vivo experiments enables comparable biologic interactions between the imaging molecule and the protein of interest, which may increase the reliability of experiments during the drug discovery process.

In summary, the availability of InSphero AG 3D microtissues on a large scale allows a completely new generation of screening applications that can be run on the Operetta system. The applicability of in vivo NIR dyes to this new model system allows the quantification of physiologically relevant readouts and will help to improve the predictive power of preclinical experiments.

1. Drewitz, M. et al. Towards automated production and drug sensitivity testing using scaffold-free spherical tumor microtissues. Biotechnol. J. 6, 1488-1496 (2011).

2. Conus, S. \& Simon, H.-U. Cathepsins and their involvement in immune responses. Swiss Med. Wkly. 140, w13042 (2010); doi:10.4414/smw.2010.13042.

3. Choi, J.Y. et al. Overexpression of MMP-9 and HIF-1a in breast cancer cells under hypoxic conditions. J. Breast Cancer 14, 88-95 (2011).

4. Nelson, A.R. Matrix metalloproteinases: biologic activity and clinical implications. J. Clin. Oncol. 18, 1135-1149 (2000).

5. Harris, A.L. Hypoxia- a key regulatory factor in tumour growth. Nat. Rev. Cancer. 2, 38-47 (2002).

6. Dai, Y. Bae, K. \& Siemann, D.W. Impact of hypoxia on the metastatic potential of human prostate cancer cells. Int. J. Radiat. Oncol. Biol. Phys. 81, 521-528 (2011).

7. Lin, R.-Z. \& Chang, H.-Y. Recent advances in three-dimensional multicellular spheroid culture for biomedical research. Biotechnol. J. 3, 1172-1184 (2008).

This article was submitted to Nature Methods by a commercial organization and has not been peer reviewed. Nature Methods takes no responsibility for the accuracy or otherwise of the information provided. 
